\title{
支配大白鼠中缝大核区的单胺能神 经末梢的来源
}

\author{
董新文沈揋 \\ (中国科学院上海生理研究所)
}

\section{摘要}

本文应用逆行荧光物标记法和荧光组织化学的结合技术研究了大白鼠中煡大核 (NRM) 区的单胺能神经末梢的来源。结果证明，支配 NRM 的神经末梢来源于去 甲肾上腺素 (NA) 能、5-羟色胺 (5-HT) 能和非单胺能三类神经元。NA 能末梢 主要起始于延脑网状外侧核 (A 1 区)、下檄榄核背外侧区 (A 3 区)、桥脑蓝斑核（A６ 区) 的腹部、蓝斑下核 (A7 区) 和中脑中㖓背核 (B7 区) 外侧区的 NA 能细胞. 5-HT 末梢主要发自于延脑中㖓苍白核 (B1 区)、中琏喑核 (B2 区)、桥脑中缝核 (B5-B6 区) 和中脑中缝核 (B8-B9 区) 的少量 5-HT 细胞. 非单胺能卡梢主要来源于下橄 榄核和脑干各个水平的网状结构，如延脑巨细胞网状核、桥脑网状尾核和中脑背被盖 区腹外侧的神经细胞. 对这些未梢可能的功能进行了讨论.

自从 Dahlström 和 Fuxe ${ }^{[1]}$ 用菼光组织化学方法揭示 NRM 含有大量的 5-HIT 能神经元 以来,有关这个核的结构和功能的研究日益增多。已有的工作确认，NRM 在吗啡、电刺激脑 和电针刺激所产生的镇痛中起着重要的调节作用 ${ }^{[2-4]}$. Fuxe $e^{[5]}$ 又首先证明, NRM 的 5-HT 能 神经元接受丰富的 NA 能末梢的支配. 但到目前为止,对于这些末梢的来源和功能意义很少 了解. 虽然有人用辣根过氧化物醮 (HRP) 追踪过 NRM 及其邻近区的传入联系，但由于末 能与组织化学方法结合,而不能鉴定标记细胞的化学性质.

Björklund 和 Skagerberg ${ }^{[6]}$ 采用 Kuypers 等 ${ }^{[7]}$ 发展的逆行茨光物追踪法和茨光组织化学 的结合妓术,能同时确定单胺能神经元的化学性质和追踪这些神经元的纤维投射,为研究单胺 能神经元的联系提供了理想的方法. 本实验的目的是采用这种结合技术追踪支配 NRM 区单 胺能末梢的来源.

\section{一、材 料 和 方 法}

实验动物为成年大白鼠,共 27 只, 体重都在 $250 \mathrm{~g}$ 左右. 月成巴比妥麻醉后 $(40 \mathrm{mg} / \mathrm{kg}$ 体重), 将大白鼠固定于定位仪上, 参照 Skinner 的鼠脑图谐 ${ }^{[8]} P=6.5 \mathrm{~mm} ; L R=0 \mathrm{~mm} ; H=$ $7.8 \mathrm{~mm}$ 。通过 $1 \mu \mathrm{l}$ 的微量注射器, 用压力注射法向 NRM 区微量注射逆行苂光物快蓝（Fast 


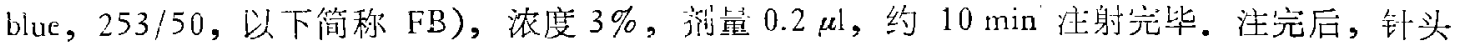
在脑内原位滞留 $10 \mathrm{~min}$ ，以防 $\mathrm{FP}$ 液外溢。手术在消瑟条件下进行，术后给动物泩射青露素， 防止伤口感染. 动物任活 2--3天, 然后快速断头致死, 开顷取脑, 讱取延脑、桥脑和中脑, 按改 艮的单胺茨光组织化学方法 ${ }^{[9]}$ 制作切片. 制片步聚大致上 Björklund 和 Skagerberg 介绍的 逆行菼光物标记法和单胺苂光冰冻干燥法的结合技术相似. 切取的脑组织任液氮冷却的开戊

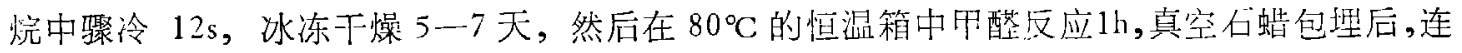
续切片, 片厚 $10 \mu \mathrm{m}$. 在 Zeiss 苂光显微镜下, 用两种滤光片系统分别观察同一均片上的单胺

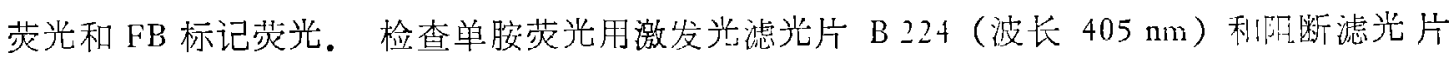
$\mathrm{G} 247$ (波长 $580 \mathrm{~nm}$ ). FB 苂光用激发光渍片 U204 (波长 $360 \mathrm{~nm}$ ) 和阻断滤片 $\mathrm{G} 243$ (波长 $480 \mathrm{~nm}$ ) 观察. 两种茨光分别照像, 以资比较.

\section{二、观 察}

\section{FB 注射部位的苂光特征}

图版 $I, 1$ 是在面神经运动核水平, FB 注人 NRM 的显微镜照像. 从图中看出, FP恰好 注射在 NRM 区的中心位置, 注射位置呈圆形或卵圆形,整个注射范围发射出明亮的苂光, 按 苂光颜色的不同, 可将整个注射区分为三个环形带. 内带或中心带呈强寅苂光（图版 I, 1 中 a), 带内组织坏死, 其直径约 $0.35-0.5 \mathrm{~mm}$; 中间带或中心外带(图版 I, 1 中 b) 发射出明亮 的淡黄到银白色的菼光, 直经约 $0.5-0.8 \mathrm{~mm}$, 带内组织含有胶质细胞的疤痕. 外带或扩散带 范围最大, 直径约 0.8-1.5 mm, 呈现出明亮的监色荧光, 带内组织基本上正常(图版 I, 1 中 c). $\mathrm{FB}$ 的注射范围与其浓度和剂量有关, 也可能和脑组织的致密或疏松程度有关。本实验只 观察 FB 注射位置局限在 NRM 区的脑7组织.

\section{2. 单胺荧光和 FB 标记苂光的鉴别}

由于单胺苂光和 $\mathrm{FB}$ 标记荻光的颜色和细胞学特征不同, 在苂光显微镜下, 用不同的滤光 片系统照明 (详见材料和方法)，很容易区别同一切片上的含 FB 和不含 FB 的单腚能细胞. 不 含 FB 的单胺能细胞在 B 224 和 G 247 滤光片系统的照明下, NA 的苂光为黄-绿色(图版 II, 1 , 箭头所指示的细胞)； $5-\mathrm{HT}$ 呈黄色(图版 II, 3, 䇽头所指示的细胞). 两种单胺能细胞的 细胞核都无荧光, 胞质和突起中苂光颜色均匀, 明亮度基本一致. 细胞质和突起无苂光颗粒, 突起一般不长。实际上, 用观察单胺苂光的㵂光片系统, 可以同时鉴别 FB标记和非 FB标记的 单胺能细胞. FB 标记的单胺细胞, 除细胞质和突起内具有单胺固有的荧光之外, 细胞质中还 有许多明亮的 FB 标记的黄色颗粒,细胞核专围有明显的黄苂光晕. 参看图版 II, $1,3,5$ 不业 符头的大多数细胞,比较图版 II, 1,3 中带箭头和不带箭头的两种细胞, 可容易地将 FB 标记的 (无箭头) 和非 FB 标记(带箭头) 的单胺细胞区别开来. 用 U204 和 G243 滤光片系统检查同 一切片上的非 FB 标记细胞(图版 II，2，4，6) 时, 单胺苂光消失或明显减弱, 只能看到 FB 标 记特征的细胞,这些细胞的胞质和树突的背景荧光呈蓝绿色, 散布在胞质和树突中的FB标记 颗粒放射出银白色的荧光,核周围有耀眼的银白色的菼光环或苂光晕,可看到的细胞突起一般 都比非 FB 标记的单胺细胞长, 核苂光很弱或无楚光 (图版 $11,1,3,3$ 中箭头所指示的单胺细 胞在图版 II, 2, 4, 6 中不再出现或菼光很弱).

\section{FB 标记细胞的分布}


在 NRM 注射 FB 之后, 被 FB 标记的细胞广泛地分布于脑干的各个区,这些细胞包括 NA 能、5-HT 能和非单胺能三类. 图 1 概括了各类 FB 标记细胞的分布区.

(1) FB 标记的 NA 能细胞的分存

一般认为,脑下尾湍(主要指延一桥脑) 各个含儿茶酚胺 (CA) 的细胞区属于: NA 能细胞 群。按 Dahlström 和 Fuxe 的命名 ${ }^{[1]}$, 这些细胞区应包括 A 1-A7 细胞群. 在 NRM 注射 FB 之后，延脑网状外侧核 (AI 区) 中大多数 NA 能细胞被 FB 标记 (图版 II，1，2 和图 1，中 1 A 1)，标记细胞的数量约占 A 1 区 NA 细胞总数的 80-99\%，这些标记细胞主要位于延脑 网状外侧核区的体部，胞体圆形或卵圆珍，多为双极或多极形细胞。胞体中等大小，平均直径 约 14--19 $\mu \mathrm{m}$. 非 FB 标记的 NA 细胞(图版 II，I 箭头)多见于 A1 区的背外侧和腹内侧 部. 除 A 1 区之外,在延脑下橄榄核背外侧区,相当于 A3 区 (图 1, 中 4, A 3) 也有 FB 标记的 $\mathrm{NA}$ 细胞, 这群细胞数量少, 在横切片上, 往往只有 2-4 个细胞, 被 FB 标记的细胞数目略与 这个数字相近. 细胞的形状和大小与 A 1 区的细胞相似, 这群细胞可看作是 A 1 细胞群向乑 侧方向的延伸。在 NRM 注射 FB 之后, 桥脑内 FB 标记的 NA 细胞主要分布于蓝斑 (A6区) 和监斑下核 (Ai区)。蓝斑背部、头端和尾端的 NA 细胞很少被 FB 标记，标记细胞多集中于 监䇋的腹部(图版 II，5，6) 约占蓝斑 NA 细胞总数的 $15-25 \%$,标记细胞的胞体呈圆形或 卵圆形，大都是多极或双极型细胞，细胞中等大小，胞体直径平均为 $15-21 \mu \mathrm{m}$. 蓝斑下核 (图版 II，5,6 (SC) 和图 1, 中 6) FB 标记的 NA 细胞约占该核 NA 细胞总数的 25-35\%,这 些细胞大都是多极形, 胞体大, 平均直径约 18-23 $\mu \mathrm{m}$. 一般认为,中脑水平以上的儿茶酚胺 能细胞群属于多巴胺 (DA) 能细胞,但在中脑中央灰质腹侧、中㖓背核 (B7 区) 外侧及其邻近 区也有 NA 细胞的分布(图 1 中 8)。这些细胞的一部分也被 FB 标记. 标记细胞呈圆形或卵 圆形,胞体平均直径约 $15-21 \mu \mathrm{m}$.

综述以上观察，NRM 中的 NA 传人末梢主要来源于 A1, A3, A6 腹部, A7 和中缝背核 (B7) 外侧的 NA 能细胞. 而不来源于脑干其他 NA 细胞群 (A2, A4, A 5). 这些细胞群的 NA 细胞可能投射至 NRM 以外的核团.

(2) FB 标记的 5-HT 能神经元的分布

在 NRM 注射 FB 之后, 脑干中㖓核系统的一些结构中发现 FB 标记的 5-HT 细胞(图 1 中 $2,4,7,8)$. 这些标记细胞主要分布于延脑的中缝苍白核 (B 1)、中缝暗核 (B 2)、桥脑中缝 核 (B6、图版 II，3，4)、中脑中缝中央核(B8)和中脑内侧丘系及其周围的网状结构 (B9)中 (图 1 中 8). 在横切片上, B1 区的 5- HT 细胞约 5-7 个,其中 1-2 个细胞被 FB 标记,略占 5-HT 细胞总数的 20-30\%,标记细胞常出现于 B1 区背侧或中间部,腹侧没有标记细胞. 中 缝暗核内 FB 标记细胞比较多,约占 5-HT 细胞总数的 $40-50 \%$. 这些细胞为双极或多极 形, 大都呈背腹方向排列, 细胞中等大小, 胞体平均直径约 13-17 $\mu \mathrm{m}$ 。桥脑中缝核(B5-B6) 内 $\mathrm{FB}$ 标记的 5-HT 细胞约占 5-HT 细胞总数的一半. 这些标记细胞多为圆形或梭形, 胞 体比较小, 平均直径约 9-13 $\mu \mathrm{m}$. 中脑虽是 5-HT 细胞 (B7-B9) 集中的地区之一,但 FB 标 记细胞很少。标记细胞仅出现于 B8 区的正中央和 B9 区的脚间核外侧边缘. B 7 区几乎找 不到标记细胞.

以上观察表明，NRM 除接受 NA 能未梢支配之外,还接受来源于 B 1, B2, B6, B 8 和 B9 的 5-HT 纤维的传人联系。 


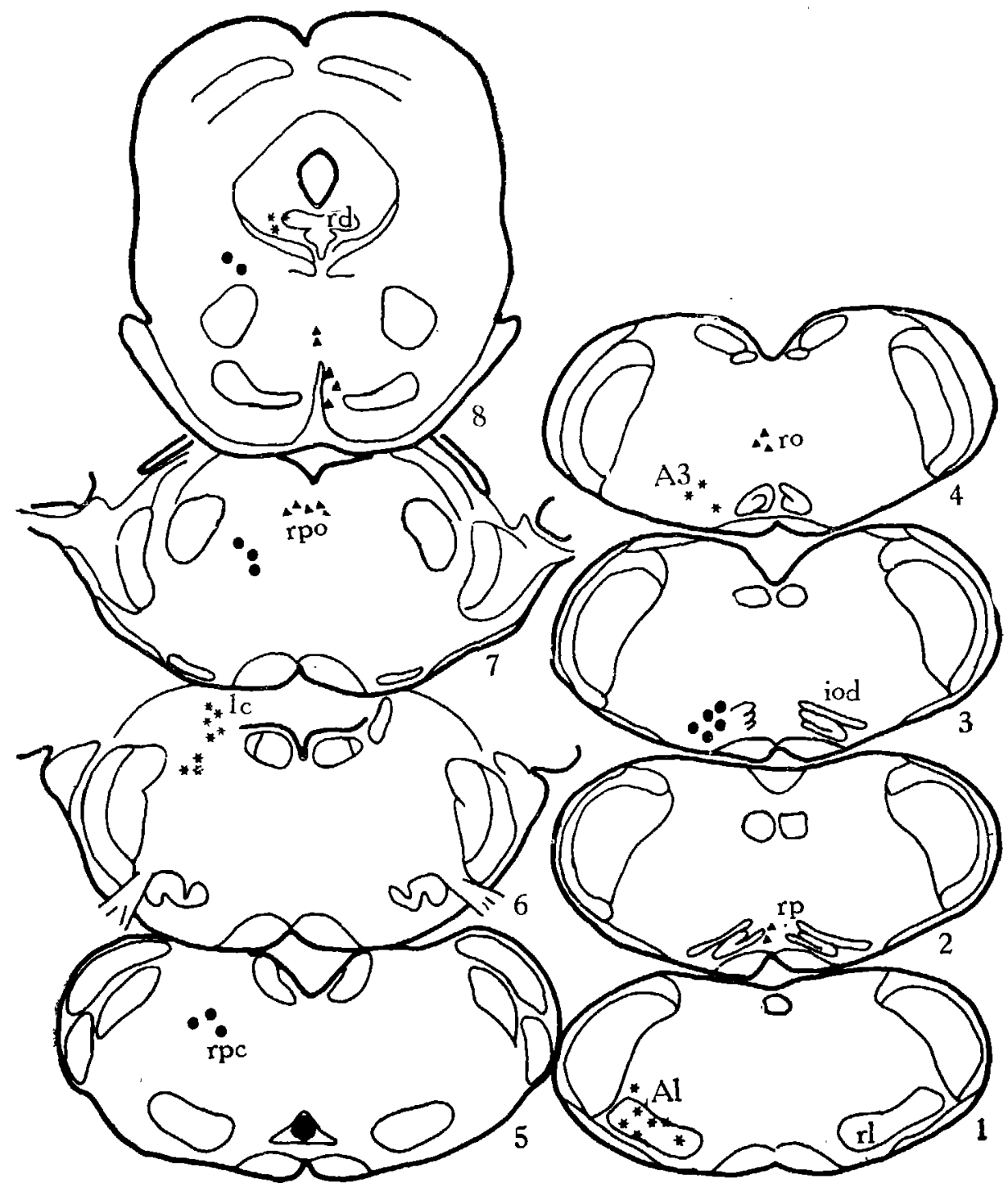

图 1 在中缝大核区注射 $\mathrm{FB}$ 之后, FB 标记细胞的分布

$(\mathrm{rl}$ ，延脑外侧网状核；rp，中琏苍白核; iod，下橄梲背核；ro，中煡暗核；rpc，延 脑网状小细胸核；lc，兰斑; rpo，桥脑中缝核; rd，中缝背核。

* 示 FB 标记的 NA 细胞 $\triangle$ 示 FB 标记的 5-HT 细胞, - 示 FB 标记的非单胺能细胞)

(3) FB 标记的非单胺能神经元的分布

在 $\mathrm{NRM}$ 注人 $\mathrm{FB}$ 之后, $\mathrm{FB}$ 标记的非单胺能神经元主要分布于下橄榄复合核和脑干各个 水平的网状结构(图 1,中 $3,5,7,8$ ). 在下橄榄复合核中,标记细胞主要集中下檄榄核,副橄榄 背核和副橄榄内侧核标记细胞很少. 延脑网状结构中, 巨细胞网状核背外侧区的标记细胞较 多（图版 I，2），这些细胞的胞体大,平均直径 18-24 $\mu \mathrm{m}$ ，标记的突起很长，有时还能看到 突起分枝 (图版 I，2 箭头)，细胞大都是多极形。胞质和突起中布满着银白色的标记颗粒,胞 垓围的银白色荻光环很明亮。延脑网状结构的其他区域中只有零星的散在的标记细胞，似 
无明确的定位分布. 桥脑网状结构的 FB 标记细胞常见于脑桥尾部网状核, 标记细胞数量少 (图版 $\mathrm{I}, 3$ ). 在中脑, FB 标记细胞常分散于中脑中央灰质腹部外侧的边缘及其邻近区. 标 记细胞数目少,在每个横切片上约 3-4 个.

\section{三、讨 论}

\section{1. 方法学问题}

自从 Kuypers 等 ${ }^{[7]}$ 发展逆行荻光物追踪神经通路的方法之后, 利用此方法和递质组织化 学 (荧光组织化学、免疫组织化学、酶组织化学)的结合技术相继出现. 这些技术正在为神经系 统的化学解剖学作出贡献. 前面提到，Björklund 和 Skagerberg ${ }^{[6]}$ 首先报道了同时用逆行荻 光物标记法和苂光组织化学技术研究单胺能神经元的投射. 他们试验了包括 $\mathrm{EB}, \mathrm{Bb}, \mathrm{DAPI}$, $\mathrm{TB}, \mathrm{GB}, \mathrm{PI}$ 等多种逆行苂光物,这些苂光物都能成功地与单胺苂光组化方法相结合. FB 是 后来新发现的逆行菼光物之一种 (Bentivoglio 等 ${ }^{\left[{ }^{0]}\right]}$ ), 在过去 Björklund 和 Skagerberg 的试验 中未能采用. 本实验证明, $\mathrm{FB}$ 和其他逆行苂光物一样,也能很满意地和苂光组化结合, 而不丧 失其苂光亮度. 这种结合方法步骤简单,在脑内注射 FB 之后,标本完全按茨光组化冰冻干燥 法处理, 不需增加任何其他步骤, 单胺和 FB 两种荻光能同时显示, 并可制作连续切片. 和单 独的 $\mathrm{FB}$ 逆行荻光物追踪法相比, FB 和苂光组化结合后, 由于组织经过聚冷和冰冻干燥等步 骤, 茨光更加清晰、明亮, 在苂光显微镜的紫外光照射下,标本可重复检查, 而不致使苂光消褪. 切片可保存好几个星期. 但 $\mathrm{FB}_{\mathrm{i}}$ 对单胺苂光稍有削弱或抑制的影响, 这和双标记或三标记苂光 之间的相互抑制现象是相似的. 经验表明，逆行荧光物标记法比HRP 法容易与荧光组织化 学结合, 是研究单胺神经元的精细投射，包括分枝投射的理想方法之一. 从我们的实验结果 看，逆行茨光物与荻光组化冰冻干燥法的结合是值得推荐的.

\section{2. 关于 NRM 中 NA 能传人纤维的来源和功能}

对于 NRM 及其邻近核团的传入联系，以前有过数篇报道 ${ }^{[11-13]}$. Takagi 等在老鼠 NRM 注人 HRP 之后,曾明确指出,支配 NRM 的 NA 能木梢来源于 A 1 和 A 3，而不来源于 A6 或其他含 $\mathrm{NA}$ 的细胞群. 实际上,他们的结论是推测性的. 因为 $\mathrm{A} 1$ 和 $\mathrm{A} 3$ 区含 $\mathrm{NA}$ 细细是 众所周知的,所以在 NRM 注人 HRP 之后, 这两个区中出现的许多标记细胞可以认为是 NA 能的. 我们的实验用组化方法证实标记细胞确属 NA 能的. 此外, 我们还观察到, 除 Al 和 A3 之外，NRM 还接受 A'6 腹部、A7 和中㖓背核外侧区的 NA 能细胞的传人未梢. Abols 和 Basbaum ${ }^{[12]}$ 在猫的 NRM 注射 HRP 之后,发现 A6 和 A7 均有少量标记细胞. 我们的观察 进一步肯定这些细胞是 NA 能的. 我们估计, $\mathrm{A} 6$ (主要是腹部) 和 A 7 被 FB 标记的 NA 细胞 约分别占两个核中 NA 细胞总数的 $15-25 \%$ 和 $25-35 \%$ ，其中不被标记的细胞占多数。

至于 NRM 中 NA 能末梢的功能, 目前知道得很少. 我们曾用 6-稚基多巴胺 (6-OHDA) 损毁 NRM 中的 NA 未梢后, 观察到大白鼠电针镇痛效应提高 ${ }^{[14]}$. Hammond 等 ${ }^{[15]}$ 报道, 在 NRM 微量注射 NA 的拮抗剂之后,大白鼠疼痛减弱. 这些资料提示, NRM 区的 NA 能 末梢参与痛党的调节作用. 鉴于一般认为, NRM 中的 5-HT 神经元与镇痛有关. 因此, 我 们推测, 支配 NRM 中的 NA 末梢可能对其中 5-HT 能神经元的镇痛作用有牒制性影响. 本 实验证明, NRM 中的 NA 纤维来源于多个含 NA 能细胞群, 是否这些细胞群的全部或一 部分参与 NRM 的痛觉调节作用, 尚待进一步研究. 


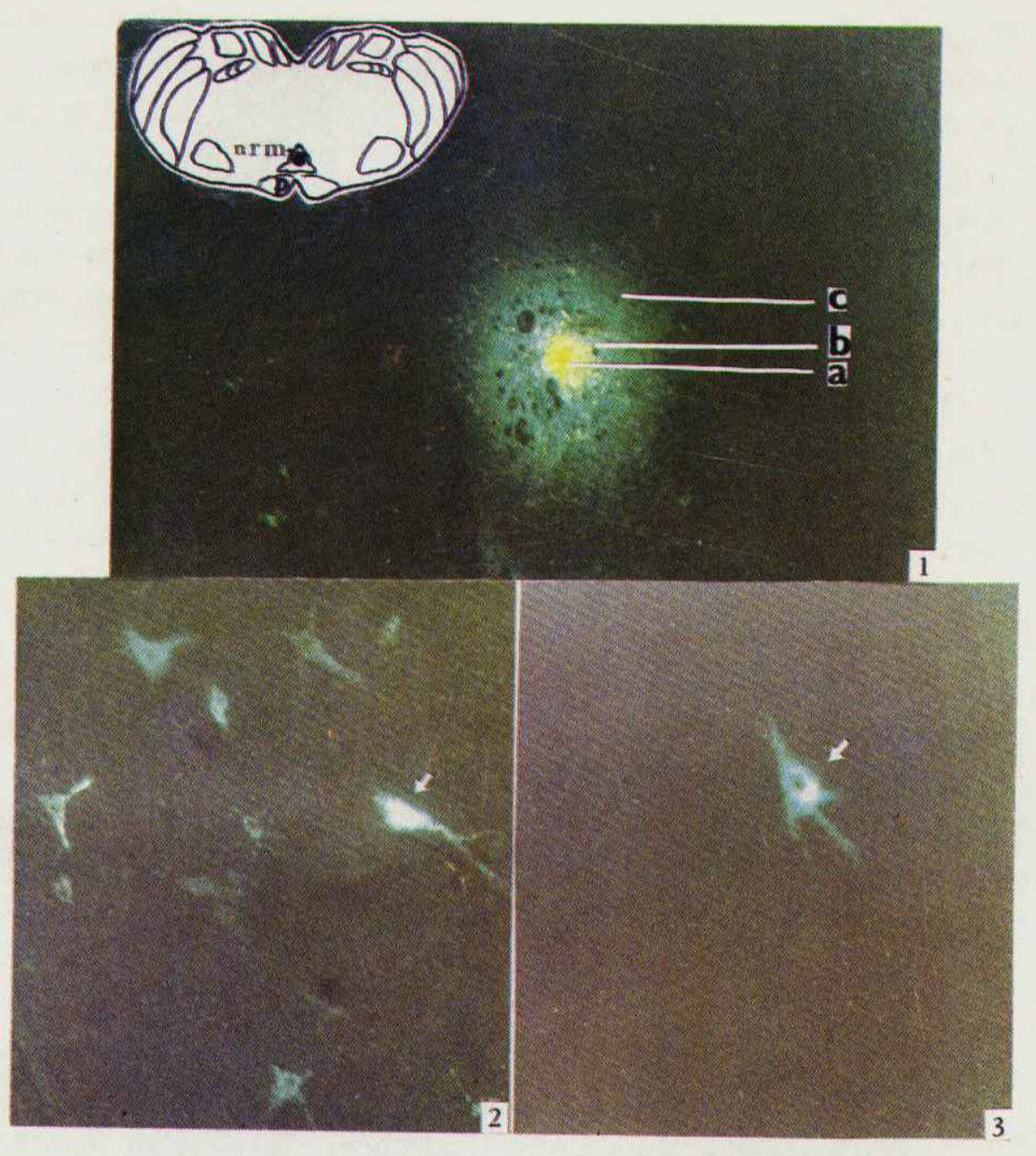

1. 在大白鼠中琏大核 (NRM) 区注射 FB 的显微镜照像。nrm, 中琏大核。注射区可分内带 (a), 中间 带 (b) 和外带 (c) $\times 216 。 2,3$. 分别为延脑和桥脑网状结构中 FB 标记的非单胺能细胞 (滤光片 $360 \mathrm{~nm} / 480 \mathrm{~nm}$ ) $\times 216$ 。 


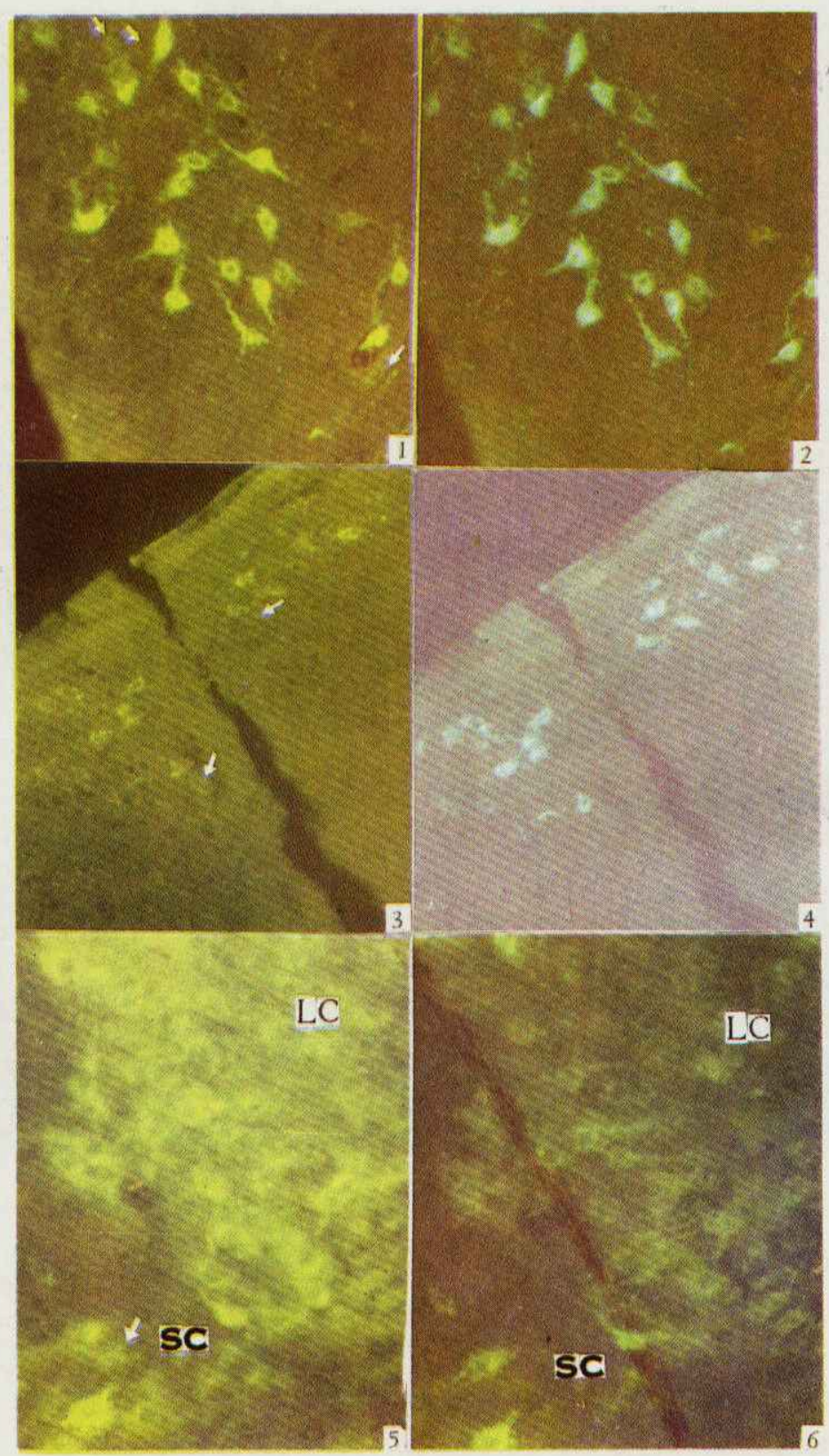

在大白瓬中琏大核区注人 FB 之后,在同一切片上可同时观察到单胺荧光和 FB 标记苂光

$(1,3.5$. 滤光片 $405 \mathrm{~nm} / 580 \mathrm{~nm} ; 2,4,6$, 滤光片 $360 \mathrm{~nm} / 480 \mathrm{~nm}) 。 1,2$ 为延脑外侧网状核, 箭头指示 未被 $\mathrm{FB}$ 标记的 NA 细胞, $\times 189 ； 3,4$ 为桥脑中琏核, 箭头表示未被 $\mathrm{FB}$ 标记的 $5-\mathrm{HT}$ 细胞 $\times 189 ; 5,6$ 为桥 脑蓝斑核 ( LC) 和蓝斑下核 ( SC), 注意 LC 中被 FB 标记的细胞主要分布于 $\mathrm{LC}$ 的腹部, $\times 189$ 。箭头指示 $\mathrm{SC}$ 中未被 FB 标记的 NA 细胞, LC 中大量未被 FB 标记的 NA 细胞主要分布于 LC 的背部, 这些细胞未一一用 箭头指出. 


\section{3. 关于 NRM 中 5-HT 纤维末梢的来源和功能}

NRM 中是否存在 5-HT 能传入末梢? 过去是不清楚的. 其主要原因是, 荻光组织化学: 方法对 5-HT 神经元的显示不及儿茶酚胺能神经元灵敏。加之，NRM 区的 NA 能末梢很稠 密,荻光很强, 掩盖了弱装光的 $5-\mathrm{HT}$ 末梢的存在. 本实验在 $\mathrm{NRM}$ 注射 $\mathrm{FB}$ 之后, 在尾中㖓 核、桥脑中缝核和中脑中缝核系统中都发现 FB 标记的 5-HT 细胞, 间接地证明了 NRM 中 5-HT 纤维末梢的存在. 利用 HRP 和免疫组织化学结合, Beitz ${ }^{[16-17]}$ 最近观察到,传人 NRM 的纤维有 5-HT,P 物质、脑啡肽和神经降压素 4 种. 其中 5-HT 纤维主要来源于中脑 B8 和 B9 细胞群, 我们虽在 B8 和 $\mathrm{B} 9$ 区看到 $\mathrm{FB}$ 标记细胞, 但标记细胞数目不多. Beitz 进一步讨 论到,传入 NRM 的 5-H'T 末梢与内源性镇痛机制有关.

由于 $N R M$ 与 $B 1$ 和 $B 2$ 邻近, 我们曾怀疑, $B 1$ 和 $B 2$ 区出现的 $F B$ 标记的 5-HT 细 胞可能由于 FB 扩散所致. 但仔细检查 $\mathrm{FB}$ 的注射痕迹(图版 $I, 1$ ) 和标记细胞的数量和分布之 后，我们的怀疑减少了. 例如, 中缝苍白核与 NRM 最接近,但标记细胞极少, 而远距离的桥 脑中琏核标记细胞很多,这说明 $B 1 、 B 2$ 的标记细胞不是扩散造成的. 但是,由于注射而损伤 的过路纤维摄取 FB 的可能性是难以排除的. Cummings 和 Felten ${ }^{[18]}$ 用 Golgi 等多种方法证明, 延脑存在着中煡树突束 (Dentrite Bundle). 在 NRM 注射 FB 之后, 我们倾向于 B1 和 B2 的部分 FB 标记细胞可能是通过树突束摄取 FB 之后而运输到标记细胞体的. 延脑尾中缝核 通过树突束相互联系,可能参与运动、内脏活动和神经内分泌的调节作用.

最后, 我们知道, NRM 含有多种神经递质的神经元, 除 5-HT 能细胞之外, 还有胆碱 能 ${ }^{[19]}$ 和多肽能细胞 ${ }^{[20]}$; 有些细胞还有 $5-\mathrm{HT}$ 和 P 物质或促甲状腺释放激素共存 ${ }^{[20]}$. 结合本 实验和 Beitz 的观察, 支配 NRM 的神经末梢也是含不同神经递质的, 至少有 NA 能、5-HT 能和多肽能(如 $\mathrm{P}$ 物质、脑啡肽、神经降压素) 等. NRM 的复杂结构为其复杂功能提供了形态 学基础.

\section{参考 文 献}

[1] Dahlstrom, A and Fuxe, K., Acta physiol. Scanz. Suppl, 232(1964), 1-55.

[ 2 ] Proudfit, H. K, Neuroscicence, 6(1981), 945-951.

[ 3 ] Pomeroy, S. L. and Behbehani, M. M., Brain Res., 176(1979), 143-147.

[ 4] 董新文, 蒋芝华, 生理学报, 33(1981)，24--29.

[ 5$]$ Fuxe, K., Acta Physiol. Scand. Suppl, 247(1965), 38-85.

[ 6 ] Bjorklund, A. and Skagerberg, G., J. Neurosci. Meth., 1(1979), 261-277.

[ 7 ] Kuypers, H. G. J. M. et al., Neurosci. Jett., 6(1977), 127-135.

[ 8 ] Skinner, J. E. Neuroscicence: A Laboratory Manual, Saundets, W. B. Company, 1971.

[ 9] 望新文、蒋芝华, 生物化学与生物物理学报, 13(1981)，117-120。

[10] Eentivoglio, M. et al., Neurosci. Lett., 18(1980), 25-30.

[11] Gallagher, D. W. and Pert, A., Brain Res., 144(1978), 257-275.

[12] Abols, I. A. and Basbaum, A. I., J. Comp. Neurol, 201(1981), 285-297.

[13] Takagi, H. et al., Brain Res., 193(1980), 315-337.

[14] 董新文等; 中国药理学报, 3(1982), 5-8.

[15] Hammond, et al., Pain., 9(1980), 85-92.

[16] Bertz, A. J, J. Neuroscience, 7(1982), 2753-2768.

[17] Beitz, A. J., J. Neturosci., 2(1982), 829-842.

[18] Cummings, J. P. and Felten, D. L., J. Comp. Neurol., 183(1979), 1-24.

[19] Tohyama, M. es al., Acta Histochem. Cytochem., 12(1979), 184.

[20] Johansson, O. et al., Neuroscience, 6(1981), 1857-1881. 